\title{
Editorial
}

\section{Calidad y control en las publicaciones}

\author{
Quality and control in \\ publications
}

Analia Veitz-Keenan ${ }^{1, a}$

${ }^{1}$ New York University, College of Dentistry, Department Oral Maxillofacial Patology and Medicine. New York, USA.

a DDS. Clinical Professor

Correspondencia:

Analia Veitz-Keenan

Correo electrónico: av244@nyu.edu

345 East 24th Street, room 829S NYU NY 10010, USA.

Fecha de recepción: 09/07/18

Fecha de aceptación: 02/08/18
La Odontología basada en evidencia es un concepto establecido hace mucho tiempo y actualmente es enseńado y practicado mundialmente. Todo comenzó desde que el concepto de la Medicina basada en la evidencia fue desarrollado y aceptado también por la Odontología. Profesionales de la salud actualmente se expresan a favor o en contra del concepto con mucho o poco conocimiento de lo que es la práctica médica basada en evidencia, pero no es el tópico central de este editorial.

La definición para la medicina ${ }^{1}$ y la adoptada por la Asociación Dental Americana ${ }^{2}$ incluye tres importantes conceptos: el uso de la mejor evidencia, la experiencia y las necesidades o preferencias de los pacientes que combinados tienen el propósito de ayudar a una decisión clínica.

¿Qué significa la "mejor evidencia"? ¿Todo lo que se publica tiene la calidad necesaria para ser utilizado o aplicado en la práctica diaria? El acceso a información encontrada en la internet y en bases de datos de la especialidad nos ayuda a encontrar información con mucha más facilidad, pero en esa cantidad de información se debe discernir entre lo que es válido y si hay relevancia para ser adoptada.

Profesionales de la salud, especialmente los que están vinculados con la educación y estudiantes de posgrado están comprometidos a presentar estudios y publicaciones para mantener las actividades académicas requeridas anualmente para promociones o graduación. Con toda esa demanda, es abrumadora la cantidad de publicaciones que van apareciendo. La realidad es que estamos acosados con la información que recibimos día a día, esa enorme cantidad de información afecta conjuntamente a pacientes y a profesionales. El tiempo necesario para leer toda la información de estudios que se publican a diario hace imposible conocerlo todo, así como entender el vocabulario técnico que se usa puede ser un obstáculo para muchos.

Actualmente a los estudiantes en diferentes universidades se los capacita para acceder y entender la calidad de estudios de todo tipo pero especialmente se los prepara para entender y poner en práctica los resultados de estudios en distintos tópicos.

Los valores estadísticos nos pueden desconcertar, si no se tiene un mínimo conocimiento de estadística y su interpretación en la práctica. Es importante para todos entender las investigaciones asociándolos con calidad de los estudios y conceptos mínimos de estadística.

Las revisiones sistemáticas son populares por combinar estudios que comparten el mismo tópico, pero la calidad de las revisiones también está asociada a la calidad de los estu- 
dios incluidos y la metodología usada en la revisión. Pero a pesar de que tenemos que interpretar la calidad de todo lo que leemos es importante controlar lo que se publica.

En conclusión, es esencial mantener la calidad en todo sentido porque es importante que nuestras decisiones clínicas puedan estar basadas en evidencia creíble, por eso, las editoriales deben tener un buen proceso para filtrar y revisar los estudios antes de ser publicados.

\section{Referencias bibliográficas}

1. Sacket DL, Rosenberg WM, Gray JA, Hyanes RB, Richardson WS. Evidence based medicine: what it is and what isn't. BMJ. 1996;312(7023):71-72.

2. ADA: Asociación Dental Americana [Internet]. ADA; c2017 [citado 9 jul 2018]. Acerca de la odontología basada en evidencia [aprox. 1 pantalla]. Disponible en: https://ebd.ada.org/en/about. 